\title{
Transient and synchronization behaviors of a standing-wave TA (Thermoacoustic) laser pair
}

\author{
Jun Ho Hyun*, Seung Jin Oh**, Sang Woong Shin***, Kuan Chen****

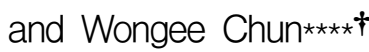

(Submit date : 2013. 10. 31., Judgment date : 2013. 11. 05., Publication decide date : 2014. 1. 14.)

\begin{abstract}
The transient and synchronization behaviors of a TA (thermoacoustic) laser pair were investigated experimentally for various crossing angles and different separation distances between the laser openings. Sound waves generated by the lasers were measured and analyzed at or near the focusing point by means of microphones, SPL meters, and a commercial software called Signal-Express. The two TA lasers were acoustically coupled through the air mass between their openings, and the only mode-locking operation that could be achieved was the one that was nearly $180^{\circ}$ out of phase. The time to achieve synchronization was found to be dependent upon the initial mistuning of the frequencies and the crossing angle between the laser axes. The synchronization process could also be accelerated by turning on the laser with the lower power input first.
\end{abstract}

Key Words : Acoustic wave, Coupled oscillators; Phase difference, Synchronization, Transient behavior

\section{Introduction}

TA (thermoacoustic) lasers have attracted increased interest in recent years due to their simple constructions and operations, as well as their potentials to use solar energy and waste heat for cleaning, materials processing, food treatment, carbon-dioxide capture, and other applications that require large quantities of inexpensive acoustic energy [1-3]. Shen et al. [4] applied solar energy to a heat source for a thermoacoustic engine and examined the thermodynamic performance at different tilted angles. $\mathrm{Wu}$ et al.[5] designed and fabricated a $1 \mathrm{~kW}$ solar-powered traveling-wave thermoacoustic electricity generation system.

$* * * * \dagger$ Wongee Chun(corresponding author) : Nuclear \& Energy Engineering Department, Jeju National University. E-mail : wgchun@jejunu.ac.kr, Tel : 064-754-3646 *Jun Ho Hyun : Nuclear \& Energy Engineering Department, Jeju National University.

**Seung Jin Oh : Nuclear \& Energy Engineering Department, Jeju National University.

\footnotetext{
***Sang Wonng Shin : Nuclear \& Energy Engineering Department, Jeju National University.

****Kuan Chen : Department of Mechanical Engineering, University of Utah
} 
They achieved a maximum electric power of $481 \mathrm{~W}$ and a maximum thermal-to-electric efficiency of $15.0 \%$.

Many studies have been carried out in the areas related to various operating parameters on the performances of TA devices and to explore their behavior for diverse applications.

Recent research interest in TA lasers was shifted to TA laser pairs and arrays. The coupling and synchronization of two TA lasers are similar to those of other coupled oscillators.

Symko et al. [6], Symko and Kwon [7], McDonald and Symko [8] have also investigated coupled TA devices. Their goal was to synchronize an array of TA prime movers using both mass and acoustic coupling. To do so, they used two TA lasers placed in a cavity in order to vary the effects of mass coupling. They found that the resulting amplitude of the synchronized arrays was the sum of the amplitudes of the individual, uncoupled engines. Chen et al [1] investigated the combination of acoustic waves generated by two identical TA lasers, and found cancellation of sound waves when the crossing angles and separation distances between the two lasers were both small .

To effectively and efficiently integrate and coordinate the operations of multiple TA lasers for enhanced acoustic energy intensity, more test results are needed. This motivated the present investigation on the transient and synchronization behaviors of TA laser pairs.

\section{Experimental setup and testing}

Fabrication and testing of the TA lasers used in the present investigation have been detailed in Chen et al.[1]. The lasers were standing-wave generators of the stack-in-a-tube type. A ceramic stack originally designed for catalytic converters was first reshaped into an octagonal cross section. Six evenly spaced grooves of $3 \mathrm{~mm}$ depth were then made on one side of the stack with a thin $\mathrm{Ni}-\mathrm{Cr}$ (Nicel-Chrome) wire fitted into the grooves. Two copper wires were inserted through the rectangular channels of the stack and their stripped ends were twisted with the $\mathrm{Ni}^{-} \mathrm{Cr}$ wire. To ensure good electrical and mechanical connections, cylindrical crimp connectors were slipped through the twisted $\mathrm{Ni}^{-} \mathrm{Cr}$ and copper wires and tightly crushed. The stack was placed inside a Pyrex glass tube approximately half way between the closed and the open end. The $\mathrm{Ni}-\mathrm{Cr}$ wire wound on one side of the stack was heated by a DC power supply. A variable resistor was connected in series with the DC power supply to adjust the power input to the electric heater of each TA laser.

As shown in Fig. 1, for a finite crossing angle between the laser axes and a separation distance (d3) greater than the laser diameter, the acoustic waves generated by the two lasers (labeled as TAL1 and TAL2) focused at a particular point and the amplitude and frequency at this point were measured and analyzed by means of a miniature microphone or a SPL meter. If the openings touched 
each other, the microphone or the SPL meter was placed at $4 \mathrm{~cm}(=\mathrm{d} 1$ in Fig. $1(\mathrm{a}))$ from the touched rims.

In the first set of experiments, TAL1 was turned on first until its frequency and amplitude reached a steady state. TAL2 was subsequently turned on and the waves generated by TAL1 triggered the standing waves in TAL2. A National Instruments DAQ card was connected to the microphone. The software Signal-Express by LabView was used to store and analyze the signals collected by the microphone. The sampling rate for the DAQ card was set to be $18 \mathrm{kHz}$ in order to maximize the number of data points collected in each cycle. The frequencyand time-domain measurements in SignalExpress were used to analyze and compare the effects of coupling of the TA laser pair for different crossing angles and separation distances. The sensitivity of the miniature microphone we used was determined to be $3.01 \mathrm{mV} / \mathrm{Pa}$ by comparing its signals to the SPL meter reading at $120 \mathrm{~dB}$. Prior to each test, each laser was powered up within a range of 20 to $35 \mathrm{~W}$ through the copper and $\mathrm{Ni}-\mathrm{Cr}$ wires. Correct wait time between two consecutive runs was crucial, as the stack initially at an elevated temperature may affect the startup behavior of the lasers. The wait time that we used was typically 60 minutes.

\section{Acoustic theories and models for a TA laser pair}

Inside the glass tube of the standing-wave
TA laser, the pressure perturbation of the fundamental mode is :

$$
\mathrm{p}^{\prime}=\mathrm{A} \cos (\mathrm{wt}) \cos [\mathrm{x} /(2 \mathrm{~L})]
$$

where $\mathrm{L}$ is the tube length and $\mathrm{x}$ is the distance measured from the closed end of the glass tube along the tube axis. At a selected point outside the TA laser pair, the sound waves generated by the two TA lasers can be expressed as [1],

$$
\mathrm{p}_{1}^{\prime}=\mathrm{A}_{1} \cos \left(\mathrm{w}_{1} \mathrm{t}\right)
$$

and

$$
\mathrm{p}_{2}{ }^{\prime}=\mathrm{A}_{2} \cos \left(\mathrm{w}_{2} \mathrm{t}+\phi_{21}\right)
$$

where A, p', w, $\phi$ correspond to the amplitude, pressure perturbation, frequency, and phase angle of the sound wave, respectively. One can use superposition to combine the waves generated by the TA laser pair,

$$
\mathrm{p}^{\prime}=\mathrm{p}_{1}^{\prime}+\mathrm{p}_{2}^{\prime}
$$

Two self-maintained oscillators, such as the pendulum clock Huygens study, or two organ pipes or TA lasers, can alter each other's frequencies enough to lock in frequency if they are sufficiently coupled through the mass between them. The mass can be that of a board on which two pendulums are mounted, or the air mass between the openings of two organ pipes or TA lasers. When the difference in uncoupled frequencies (also called detuning) is large, the coupling is 
weak and the oscillators "beat" at a frequency lower than the uncoupled frequencies of the two TA lasers, just like the acoustic oscillators studied by Spoor and Swift[9]. As detuning decreases or as the coupling increases, the beat slows down and eventually stops, at which point the two TA lasers are said to be locked or synchronized.

Presented in Fig. 2 are the calculated p' for two cosine-wave pressure oscillations of different amplitudes and frequencies. The maximum and minimum amplitudes of the combined wave are equal to the sum and difference of the amplitudes of the two waves, and the beating frequency $(=1 / \mathrm{T}$ in Fig. 2$)$ decreases as the difference between the two

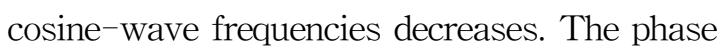
difference has no effect on the combined wave. On the other hand, if the two pressure oscillations are synchronized $\left(\mathrm{w}_{1}=\mathrm{w}_{2}\right)$, the combined wave does not beat (the maximum amplitude does not vary with time), but its maximum amplitude depends on the phase difference, changing from $A_{2}+A_{1}$ for $\phi_{21}=$ 0 to $A_{2}-A_{1}$ for $\phi_{21}=\pi$.

\section{Results and discussions}

\subsection{Onset and amplification of acoustic waves in the TA laser pair}

Compared to other types of TA prime movers such as the Stirling engine or the liquid-piston engine (a water column oscillating in a U-tube driven by the thermoacoustic effect), our standing-wave TA laser, which has one end open to the air, was fairly easy to start. As long as the electric heater maintained a high enough temperature gradient across the stack, sound waves were generated. The amplitudes of the waves increased with time until eventually the net heat input to the air oscillating in the stack was balanced by the frictional loss. Our theory is that the onset of acoustic oscillations in the laser was triggered by the background noise. Since the background noise can be decomposed into Fourier series of different wavelengths, the standing-wave design of the TA laser we tested picked up the Fourier series components of the right wavelengths (the longest one is four times the glass tube length) and amplified them.

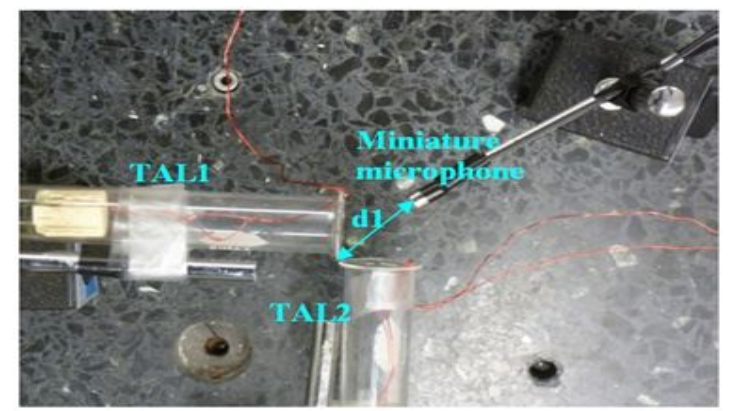

(a)

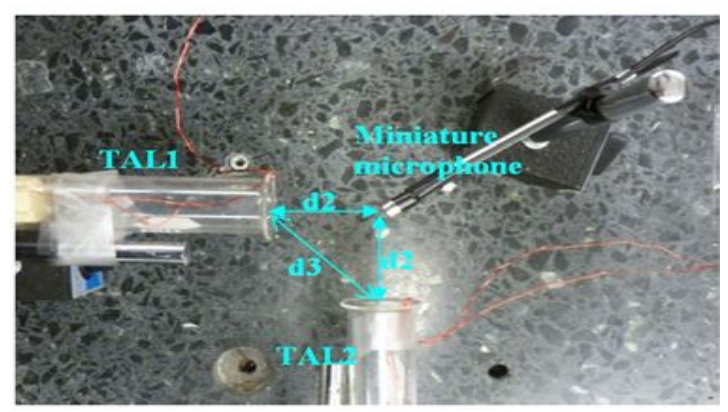

(b)

Figure 1. Experimental setup for the TA laser pair. (a) Tube openings touching each other. (b) Tube openings separated by a distance d3. 


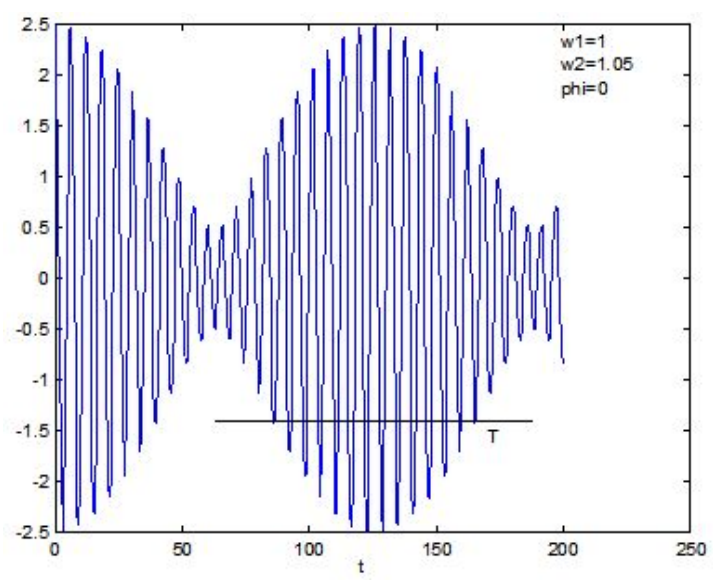

(a)

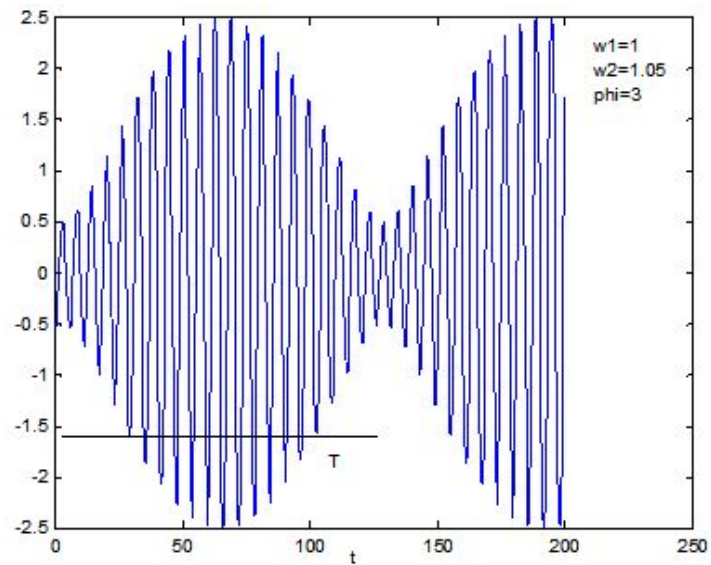

(b)

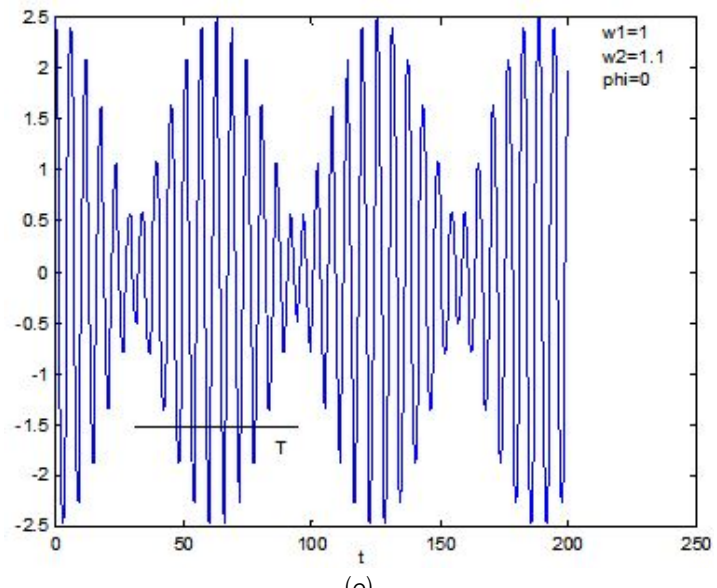

Figure 2. Calculated pressure fluctuations $p^{\prime}$ for $A_{1}=1$, $A_{2}=1.5$. (a) $W_{1}=1, W_{2}=1.05, \phi_{21}=0$ (b) $W_{1}=1, W_{2}=$ $1.05, \phi_{21}=3$ (c) $w_{1}=1, w_{2}=1.1, \phi_{21}=0$.
On the contrary, we found the piston in a TA Stirling engine or the water column in the U-tube of a liquid-piston engine we tested won't move in some cases even though the temperature gradient across the stack was higher than the one needed to sustain a steady oscillatory flow in the device. It was necessary in this case to "crank-start" the TA prime mover. This can be done by manually moving the piston of the Stirling engine back and forth, or shaking the water column in the U-tube of the liquid-piston engine. One reason such a crank start is sometimes needed for the Stirling engine or the liquid-piston engine is due to the high static friction of the piston or the water column.

Another reason may be due to the lack of an initial disturbance to the TA device which is of the right wavelength thus can be easily amplified by the thermoacoustic effect. Adding a small amount of water in the steel wool (which was used as the stack of a TA mover) of our liquid-piston engine also made it easier to start the water column oscillation. This was probably because when the water in the steel wool evaporated, there was a large expansion of the gas volume between the water column and the steel wool, triggering the initial acoustic oscillation in the tube.

Based on previous studies of the synchronization of organ pipes, it is anticipated the startup time for the standing-wave TA laser can be shortened by placing another TA laser that has reached a steady state in the close 
vicinity of the TA laser to be triggered, as shown in Fig. 1(a). Onset of acoustic oscillations can be accelerated further by increasing the crossing angle between the two TA lasers when their openings are close to each other. This is because the emitted acoustic waves remain in a planar configuration in the close vicinity of the laser opening. A large crossing angle allows more air to be pushed in and pull out of the laser to be triggered due to the displacement fluctuations at the opening of the second laser. (The strong coupling between the two TA lasers, when their openings are close to each other and the crossing angle is large, will also affect the synchronization of the TA laser pair, to be discussed in the next section.) Too large a crossing angle, however, may change the laser opening from a pressure node to a displacement node, and extinguish the sound waves in both TA lasers. This had been observed in our previous study of the TA laser pair [1]. When the crossing angle of a synchronized TA laser pair exceeded $90^{\circ}$, the planar waves exiting one TA laser struck the interior wall of the other laser, increasing the air pressure at the tube opening and changing the sound wavelengths. This is similar to the extinguishing of a single TA laser operation we observed when a large microphone was placed very close to the glass tube opening .

As the crossing angle approaches $180^{\circ}$, the planar waves exiting one TA laser will strike the interior wall of the other laser at a small angle, as shown in Fig. 3.
Air displacement fluctuations in
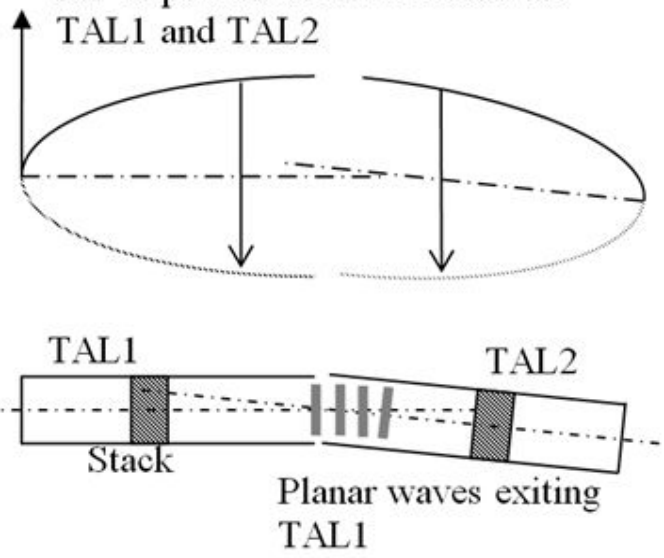

Figure 3. Air displacement fluctuations in the synchronized TA laser pair for B (crossing angle) close to $180^{\circ}$. (Planar waves exiting TAL1 strikes and reflects from the interior wall of TAL2 at a small angle)

In this case the air pressure at the tube openings deviates only slightly from the surrounding air pressure, and a synchronized operation may again be established in the TA laser pair as depicted in Fig. 3. The frequency of the standing waves will be the same as that of a single TA laser operation. The frictional loss of the TA laser pair is higher than that of a single laser operation. As a result, the power input to the electric heater must increase if only one TA laser heater is turned on, or the electric heaters of both TA lasers must be turned on [1] to sustain the standing waves plotted in Fig. 3.

\subsection{Synchronization of the TA laser pair}

The fundamental frequency of the standingwave TA laser is equal to the sound speed in the laser divided by four times the tube length. The tube lengths of the TA lasers we tested were the same. After the electric 
heater was turned on, the speed of sound in the air inside the glass tube increased as the stack and air inside the glass tube were heated by the $\mathrm{Ni}-\mathrm{Cr}$ wire. After the onset of standing waves in the glass tube, the fundamental frequency still increased slightly with time before the TA laser operation reached a steady state. The time variations of the fundamental frequencies of the two TA lasers when operated individually are presented in Table 1. In this table frequency recording started (time $=0$ ) when the acoustic waves were clearly audible. Because it was difficult to wind the $\mathrm{Ni}^{-} \mathrm{Cr}$ wire on the ceramic stack exactly the same way for the two lasers, and to control the electric power inputs to individual TA lasers very precisely, a frequency difference about $1 \%$ between the two TA laser frequencies can be seen in Table I when the two lasers operated individually. The difference in frequency disappeared when synchronization was achieved.

Shown in Fig. 4 were the measured TA laser frequencies in the process toward synchronization. The crossing angle between the TA laser axes was $30^{\circ}$ and the openings of the laser pair touched each other. In this test the second TA laser (TAL2), which has a slightly higher power input than that of TAL1, was turned on roughly 10 seconds later than TAL1. Initially the frequencies of the two lasers differed by about $2 \mathrm{~Hz}$. As shown in this plot, it took about 15 seconds for the TA laser pair to reach the state of synchronization. The amplitudes of the laser outputs are presented in Fig. 5. The amplitude of the synchronized laser pair was much lower than that before synchronization.

Table 1. Fundamental frequencies of TAL1 and TAL2 when operated individually (crossing angle $=30^{\circ}$ ).

\begin{tabular}{ccc}
\hline \multicolumn{3}{c}{ Fundamental frequencies of TAL1 and TAL2 when } \\
operated individually \\
\hline $\begin{array}{c}\text { Time in } \\
\text { seconds }\end{array}$ & $\begin{array}{c}\text { Frequency of } \\
\text { TAL1 measured in } \\
\text { Hz }\end{array}$ & $\begin{array}{c}\text { Frequency of } \\
\text { TAL2 measured in } \\
\text { Hz }\end{array}$ \\
\hline 0 & 440.68 & 443.03 \\
\hline 15 & 447.81 & 450.86 \\
\hline 30 & 452.26 & 456.28 \\
\hline 45 & 455.73 & 459.82 \\
\hline 60 & 458.16 & 461.95 \\
\hline
\end{tabular}

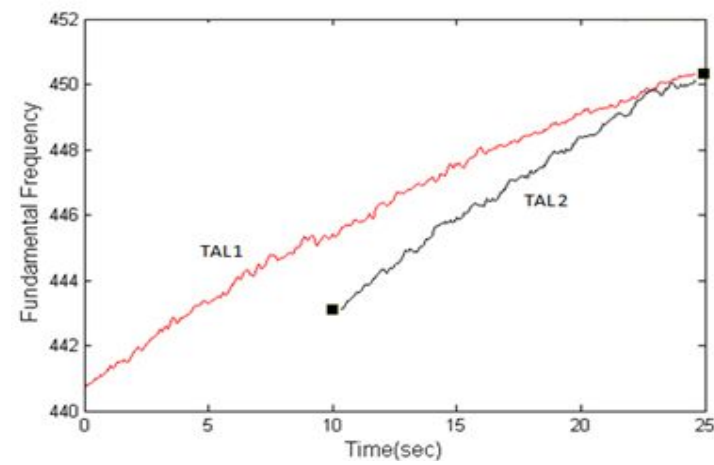

Figure 4. Measured TA laser frequencies in the process toward synchronization.

Significant drop in amplitude for a synchronized TA laser pair was also observed for $\mathrm{B}$ (crossing angle) $=90^{\circ}$, as shown in Fig. 6. In Figs. 5 and 6, the combined waves before synchronization beat at a frequency much lower than the fundamental frequencies of the two lasers, indicating slight detuning of the two laser frequencies. The measured acoustic waves were not perfectly symmetric about the $\mathrm{x}$ axis (state of zero amplitude), and the maximum and minimum amplitudes were not exactly equal to the sum and difference 
of the maximum amplitudes of the two lasers when operated individually. Comparing this observation to the theoretical results in Fig. 2 reveals some departure from simple sinusoidal waves of the two laser outputs in the process toward synchronization.

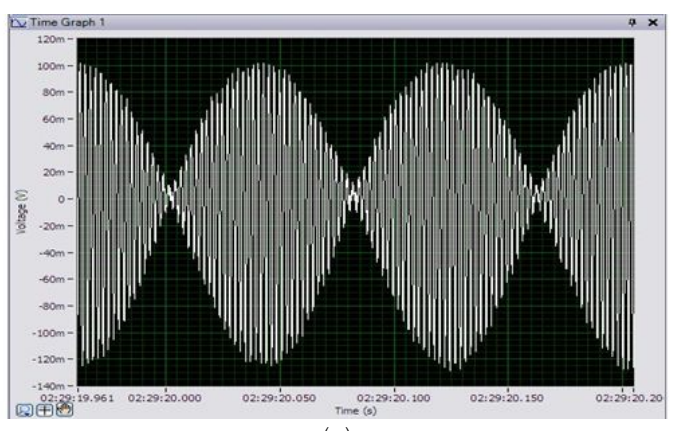

(a)

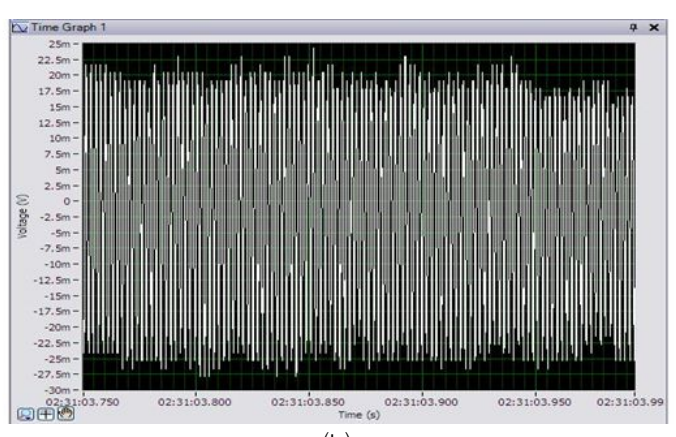

(b)

Figure 5. Combined waves of the TA laser pair with tube openings touching each other $\left(B=30^{\circ}\right.$ ). (a) Before synchorization had been achieved. (b) After synchorization had been achieved.

When the TA laser of higher power input (i.e., TAL2) was turned on first, it took much longer to reach synchronization as can be seen in Fig 7. This is because the time required for the laser of lower power input to catch up to the frequency of the one with a higher power input is longer. Thus, unless the two lasers are thermally identical, the time to reach synchronization will depend on the order in which the two lasers are turned on. To shorten the time to reach synchronization, the laser with the lower power input should be turned on first.

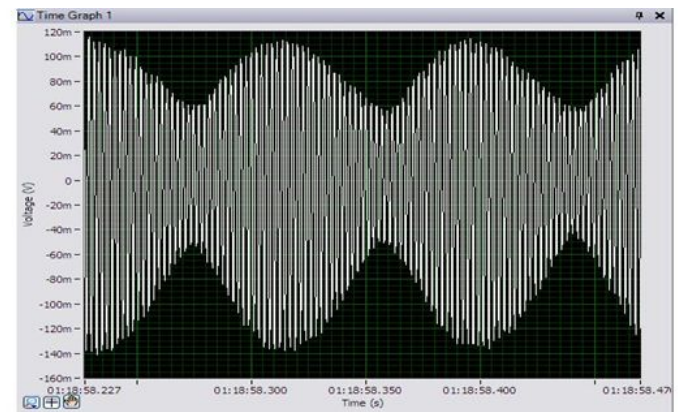

(a)

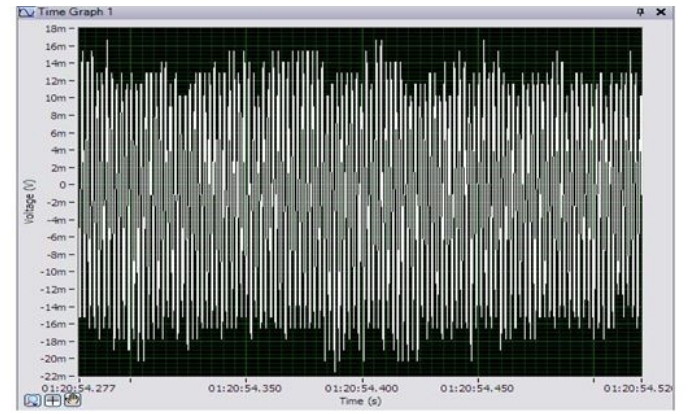

(b)

Figure 6. Combined waves of the TA laser pair with tube openings touching each other $\left(B=90^{\circ}\right)$. (a) Before synchronization had been achieved. (b) After synchronization had been achieved.

The crossing angle, B, also affects the synchronization behavior. Increasing the crossing angle makes the coupling of the two lasers stronger, thus shortening the time to reach synchronization. Shown in Fig. 8 are the sound amplitude variations for $\mathrm{B}=90^{\circ}$. Compared Fig. 8 to Fig. 7, it can be seen the time to reach synchronization was much shorter. When TAL1 (the one with the lower power 
input) was turned on first, synchronization occurred almost immediately. When the switching order was reversed, it took only about 10 seconds to reach the state of synchronization. It can also be seen clearly in Fig. 8 that the final amplitude of the combined sound waves of the synchronized TA laser pair is independent of the order in which the two lasers were turned on.

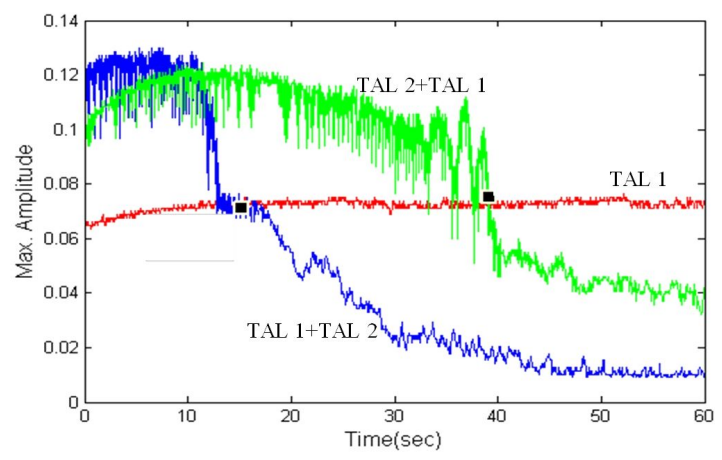

Figure 7. Variations of the maximum amplitude in the synchronization process for $B=30^{\circ}$. (TAL openings touching each other).

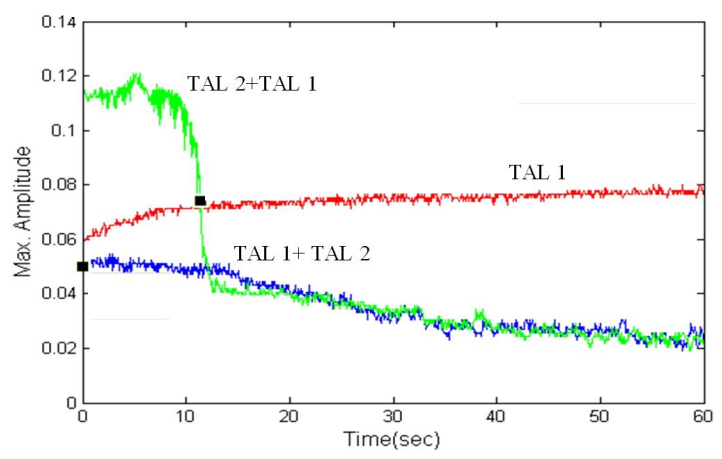

Figure 8. Variations of the maximum amplitude in the synchronization process for $\mathrm{B}=90^{\circ}$. (TAL openings touching each other)

\section{Conclusion}

Experiments were conducted to study the effects of frequency detuning and crossing angle between laser axes on the startup and synchronization of a standing-wave TA laser pair. The two TA lasers could be synchronized through the air between them if the openings of the two lasers were very close to each other. Less detuning in initial frequencies and larger crossing angles accelerated the process toward synchronization. Turning on the laser of lower power input first could also shorten the time to reach synchronization. Acoustic waves generated by the TA lasers changed from planar to spherical waves within a few diameters of the laser opening.

\section{Acknowledgments}

This work was supported by the National Research Foundation of Korea(NRF) grant funded by the Ministry of Science, ICT \& Future Planning (No. 2009-0092786)

\section{REFERENCE}

1. K. Chen, S.J. Oh, Y.J. Lee, W.J. Oh, K.H. Kim,W. Chun, Acoustic energy output and coupling effect of a pair of thermoacoustic lasers, Int. J. Energ. Res. 36 (2012) 477-485.

2. L. Zoontjens, C.Q. Howard, A.C. Zander, B.S. Cazzolato, Numerical comparison of thermoacoustic couples with modified stack plate edges, Int. J. Heat Mass Transfer 51 (2008) 4829-4840.

3. R. Surathu, A study of coupling two thermoacoustic lasers, Master thesis, University of Utah (2012).

4. C. Shen, Y. He, Y. Li, H. Ke, D. Zhang, Y. Liu, Performance of solar powered thermoacoustic 
engine at different tilted angles, Appl. Therm, Eng. 29 (2009) 2745-2756.

5. Z. Wu, W. Dai, M. Man, E. Luo, A solar-powered traveling-wave thermoacoustic electricity generator, Sol. Energ. 86 (2012) 2376-2382.

6. O.G. Symko, E. Abdel-Rahman, Y.S Kwon, M. Emmi, R. Behunin, Design and development of high-frequency thermoacoustic engines for thermal management in microelectronics, J. Microelectron 35 (2004) 185-191.

7. O.G. Symko, Y.S. Kwon, Compact thermoacoustic array energy converter, US Patent No. 8004156. (2011).

8. B.G. McDonald, O.G. Symko, Coupling of midaudio frequency thermoacoustic prime movers, J. Acoust. Soc. Am. 125 (2009) 2563.

9. P.S. Spoor, G.W. Swift, Mode-locking of acoustic resonators and its applications to vibration cancellation in acoustic heat engines, J. Acoust. Soc. Am. 106 (1999) 1353-1362. 